\title{
Técnicas de Projeto para equalizadores por subcanal para sistemas FBMC/OQAM
}

\author{
Bruno S. Chang, Wilson L. E. Lopez e Carlos A. F. da Rocha
}

\begin{abstract}
Resumo - Este artigo apresenta técnicas de projeto de equalizadores FIR por subcanal para sistemas FBMC/OQAM. As técnicas de equalização propostas usam a estimação de canal realizada no começo de cada frame transmitido. Com a resposta em frequência conhecida na posição central de cada subcanal obtém-se por interpolação de Lagrange ou geométrica pontos localizados em frequências adjacentes da frequência central. Estes pontos são utilizados para obter os coeficientes do equalizador calculados através da transformada de Fourier inversa (IFFT). O equalizador deve compensar ICI e ISI introduzidas por canais seletivos e offset temporal. Resultados de simulação demonstram a eficiência dos equalizadores.
\end{abstract}

Palavras-chave - Equalizador de subcanal, Interpolação de Lagrange, Interpolação geométrica, FBMC/OQAM

Abstract - This paper presents FIR subchannel equalizers design techniques for FBMC/OQAM systems. The proposed equalization techniques use the channel estimation obtained at the beginning of each transmitted frame. With the known frequency response at the central position of each subchannel, points in adjacent frequencies are obtained through Lagrange or geometric interpolation. These points are used to obtain the equalizer coefficients through the inverse Fourier transform (IFFT). The equalizer should compensate ICI and ISI introduced by selective channels and temporal offset. Simulation results show the equalizers' efficience.

Keywords - Subchannel equalizer, Lagrange interpolation, Geometric interpolation, FBMC/OQAM

Este trabalho foi parcialmente financiado pela Universidade Federal de Santa Catarina (UFSC), Conselho Nacional de Desenvolvimento Científico e Tecnológico (CNPq) e Coordenação de Aperfeiçoamento de Pessoal de Nível Superior (CAPES), através do projeto CAPES-COFECUB número 544/07. Bruno Sens Chang é aluno de doutorado na UFSC no Grupo de Pesquisa em Comunicações (GPqCom), bolsista CNPq, e-mail: brunosenschang@gmail.com. Wilson E. L. Lopez é aluno de doutorado na UFSC no Grupo de Pesquisa em Comunicações (GPqCom), bolsista CAPES/PEC-PG, e-mail: wenriquez@igepn.edu.ec. Carlos A. F. da Rocha é professor no Departamento de Engenharia Elétrica da UFSC, laboratório GpqCom, e-mail: aurelio@eel.ufsc.br.

\section{INTRODUÇão}

Equalização de canal para sistemas de bancos de filtros multiportadoras utilizando modulação offset QAM (FBMC/OQAM) é um tópico de pesquisa não tão bem explorado quanto em sistemas OFDM/QAM [1]. Em [1], duas estruturas de equalizadores de subcanal foram propostas para um transmultiplex. A primeira é um equalizador FIR (finite impulse response - resposta ao impulso finita) complexo de 3 coeficientes, e a segunda é uma estrutura utilizando um filtro all-pass como equalizador de fase e um equalizador de amplitude FIR com fase linear. Outras estruturas SCE (subchannel equalizer equalizadores de subcanal) foram também propostas em [25].

Diferentemente dos sistemas OFDM/QAM que utilizam equalizadores de um coeficiente por subcanal, os sistemas FBMC/OQAM, em geral, necessitam de equalizadores de vários coeficientes por subcanal para compensar canais altamente seletivos em frequência. Isto se deve ao fato de que os sistemas FBMC/OQAM não utilizam o prefixo cíclico, característico dos sistemas OFDM/OQAM.

$\mathrm{O}$ projeto de um equalizador FIR de dois coeficientes requer dois pontos da resposta em frequência do subcanal, três pontos para um equalizador de três coeficientes, e assim por diante. Portanto, uma técnica de estimação de canal mais complexa é necessária quando equalizadores de vários coeficientes por subcanal devem ser utilizados.

Neste artigo propomos um método de projeto de equalizadores de subcanal FIR baseado na amostragem em frequência e nas técnicas de interpolação de Lagrange e geométrica. O método proposto utiliza a transformada inversa rápida de Fourier (IFFT) para calcular os coeficientes de equalizadores de três e cinco coeficientes, utilizando três e cinco pontos da resposta inversa em frequência de cada subcanal, respectivamente. Baseado no conhecimento da resposta em frequência de cada subcanal na sua frequência central, os outros dois ou quatro pontos de frequências adjacentes são obtidos por interpolação, evitando assim o uso de uma técnica de estimação de canal mais complexa. 


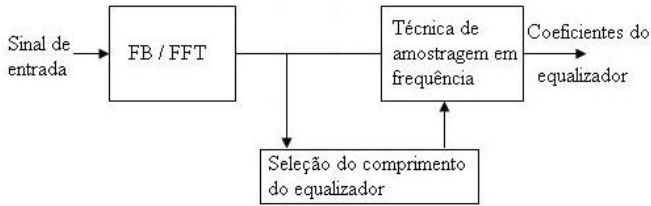

Fig.1- Projeto de um equalizador de subcanal para o receptor FBMC/OQAM

A técnica de interpolação de Lagrange é considerada adequada quando o sinal a ser interpolado apresenta um nível significativo de correlação entre suas amostras. Este é

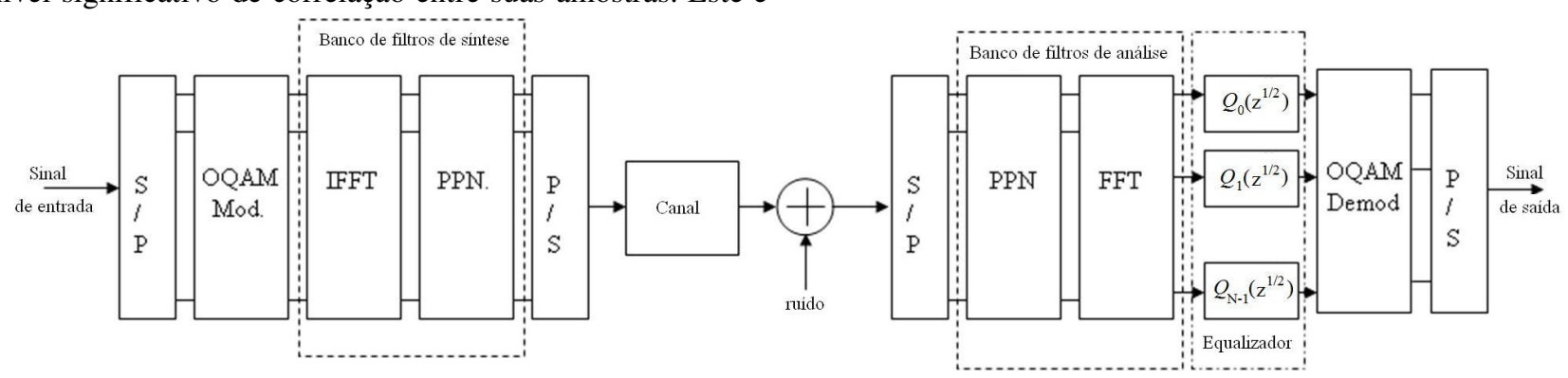

Fig. 2 - Sistema de transmissão FBMC/OQAM com equalização

Várias curvas de BER são apresentadas para comparar o desempenho dos equalizadores. Estas curvas foram obtidas utilizando o modelo de canal Veicular A [6]. Este canal foi considerado invariante no tempo durante cada burst transmitido.

Este artigo é organizado como segue. A Seção II descreve o modelo do sistema de transmissão FBMC/OQAM, mostrando o equalizador por subcanal no receptor. A técnica proposta de projeto dos equalizadores é detalhada na Seção III. Finalmente, os resultados de simulação e conclusões são apresentados nas seções IV e V, respectivamente.

\section{O SISTEMA FBMC/OQAM}

Recentemente, vários artigos [7,8] foram publicados na literatura sugerindo o uso de sistemas FBMC/OQAM (também chamados de OFDM/OQAM) como um esquema alternativo ao OFDM/QAM. As diferenças cruciais entre estes dois sistemas vêm do fato que os sistemas FBMC/OQAM usam a modulação offset QAM para cada subportadora, associada com uma estrutura de banco de filtros e o uso de uma formatação eficiente de pulso, bem localizada tanto no domínio do tempo quanto no da frequência. Estas características fazem os sistemas FBMC/OQAM menos sensíveis ao offset de frequência e permite a eles não utilizar o prefixo cíclico, o que leva a uma maior eficiência espectral. A formatação de pulso bem localizada permite o uso de equalizadores simples de o caso de sistemas multiportadoras, quando o número de portadoras é elevado e/ou quando o canal apresenta baixa seletividade em frequência. Por outro lado, o desempenho dos interpoladores de Lagrange é dependente da duração da resposta ao impulso do canal, como será discutido posteriormente. A interpolação geométrica é proposta com o objetivo de retirar esta dependência, ao custo de uma maior complexidade computacional. A Figura 1 mostra o método proposto.

subcanal para compensar as distorções de canais seletivos em frequência.

A Figura 2 mostra o modelo em banda base FBMC/OQAM usado em nossa simulação. O sinal de entrada $\left(a_{i}[n]\right)$ é obtido de uma constelação QPSK. Depois do conversor serial/paralelo, o modulador OQAM gera uma sequência de símbolos de entrada para o bloco IFFT como mostrado na Tabela 1 , onde $\mathrm{T}$ é a duração do símbolo e $a_{i}^{R}[n]$ e $a_{i}^{I}[n]$ são as partes real e imaginária de $a_{i}[n]$, respectivamente. Como as duas partes são processadas separadamente, note que a cada T/2 segundos uma IFFT de ordem $\mathrm{N}$ deve ser calculada.

Tabela 1 - Sinal OQPSK transmitido

\begin{tabular}{|c|c|c|c|c|c|c|}
\hline \multicolumn{2}{|l|}{$\downarrow_{n T}$} & \multicolumn{2}{|c|}{$\downarrow(n+1) T$} & \multicolumn{2}{|l|}{$\stackrel{\text { tempo }}{\longrightarrow}$} & \\
\hline$a_{0}^{R}[0]$ & $a_{0}^{I}[0]$ & $a_{0}^{R}[1]$ & $a_{0}^{I}[1]$ & $a_{0}^{R}[2]$ & $a_{0}^{I}[2]$ & \\
\hline$a_{1}^{I}[0]$ & $a_{1}^{R}[0]$ & $a_{1}^{I}[1]$ & $a_{1}^{R}[1]$ & $a_{1}^{I}[2]$ & $a_{1}^{R}[2]$ & \\
\hline$:$ & $:$ & : & $\vdots$ & $\vdots$ & $:$ & \\
\hline$a_{N-1}^{I}[0]$ & $a_{N-1}^{R}[0]$ & $a_{N-1}^{I}[1]$ & $a_{N-1}^{R}[1]$ & $a_{N-1}^{I}[2]$ & $a_{N-1}^{R}[2]$ & \\
\hline
\end{tabular}

A rede polifásica (PPN) combinada com os blocos IFFT e FFT forma o banco de filtros de síntese (SFB) e o banco de filtros de análise (AFB), respectivamente. O SFB e o AFB desempenham a tarefa de dividir a largura de banda de transmissão entre $\mathrm{N}$ subcanais iguais. Globalmente, os coeficientes do filtro formam a resposta ao impulso do filtro protótipo. Este filtro protótipo é um filtro passa-baixa real, que satisfaz o critério clássico de Nyquist. Os resultados de 
simulação apresentados neste artigo foram obtidos utilizando o filtro protótipo proposto em [9].

Os equalizadores $Q_{i}\left(z^{1 / 2}\right)$ espaçados em $\mathrm{T} / 2$ permitem a remoção da ISI e ICI causadas pelo canal seletivo em frequência $H(z)$ e restauram a ortogonalidade do sistema OQAM. Como veremos mais adiante, para um grande número de subportadoras e um canal com média distorção, um equalizador de um coeficiente é suficiente, exatamente como no caso de sistemas OFDM/QAM com prefixo cíclico. Já em canais altamente seletivos em frequência, equalizadores com mais de um coeficiente devem ser utilizados.

\section{TÉCNICA DE PROJETO DOS EQUALIZADORES}

$\mathrm{Na}$ seção seguinte, assumimos o conhecimento do canal nas frequências $k \Delta f$, onde $\Delta f$ é o espaçamento dos subcanais. Então, para o caso do equalizador de um coeficiente, os coeficientes do equalizador podem ser calculados através do critério zero-forcing como

$$
E Q(i)=q_{1 i}=1 / H(i),
$$

onde $i$ é o índice do subcanal.

\section{A. Interpolação de Lagrange}

Para um equalizador de três coeficientes do $i$-ésimo subcanal precisamos de três pontos da resposta inversa em frequência do subcanal: $E Q(i)$ e dois pontos intermediários $E Q 1$ e $E Q 2$, como mostrado na Figura 3. Estes pontos intermediários são obtidos utilizando os pontos de frequência conhecidos $E Q(i-2), E Q(i-1), E Q(i), E Q(i+1)$ e $E Q(i+2)$ através da interpolação de Lagrange.

A Figura 4 mostra os pontos da resposta inversa em frequência do canal utilizados para projetar o equalizador de cinco coeficientes. Os pontos EQ2 e EQ3 são obtidos usando os pontos $E Q(i-2), E Q(\mathrm{i}-1), E Q(i), E Q(\mathrm{i}+1)$ e $E Q(i+2)$ e os pontos $E Q 1$ e $E Q 4$ são $E Q(i-3), E Q(\mathrm{i}-2), E Q(i-$ 1), $E Q(\mathrm{i}), E Q(i+1)$ e $E Q(i-1), E Q(\mathrm{i}), E Q(i+1), E Q(\mathrm{i}+2)$, $E Q(i+3)$, respectivamente.

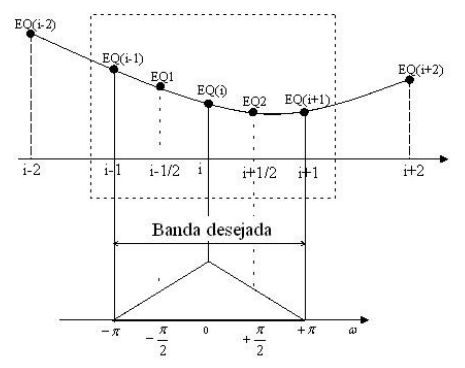

Fig. 3. Valores da resposta inversa em frequência do subcanal usados para projetar o equalizador FIR de três coeficientes.

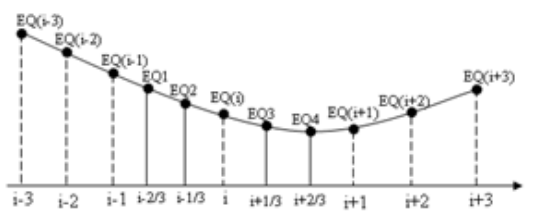

Fig. 4. Valores da resposta inversa em frequência do subcanal usados para projetar o equalizador FIR de cinco coeficientes.

No domínio da frequência, a técnica da interpolação de Lagrange corresponde a uma filtragem maximamente plana [10]. Assim, os coeficientes do filtro interpolador de Lagrange $g[n]$ são obtidos pela solução do seguinte conjunto de $2 P+1$ equações lineares:

$$
\left.\frac{d^{k}}{d \omega^{k}}\left(\sum_{n=-P}^{P} g[n] e^{-j n \omega}-e^{-j \omega D}\right)\right|_{\omega=0}=0, \quad k=0,1, \ldots, 2 P
$$

onde $|D| \leq 1 / 2$ é o atraso fracional. A solução é dada em forma explícita por

$$
g[n]=\prod_{k=-P, k \neq n}^{P}\left(\frac{D-k}{n-k}\right), n=-P, . ., 0, \ldots . P,
$$

e os valores interpolados são dados por

$$
y[m]=\sum_{n=-P}^{P} g[n] x[m-n],
$$

onde $x(n)$ é o sinal de entrada do interpolador.

Utilizando as equações (3) e (4), os pontos EQ1 e EQ2 para o equalizador FIR de três coeficientes podem ser calculados como:

$$
\begin{aligned}
& E Q 1=\frac{-5}{128} E Q(i-2)+\frac{15}{32} E Q(i-1)+\frac{45}{64} E Q(i)-\frac{5}{32} E Q(i+1)+\frac{3}{128} E Q(i+2) \\
& E Q 2=\frac{3}{128} E Q(i-2)-\frac{5}{32} E Q(i-1)+\frac{45}{64} E Q(i)+\frac{15}{32} E Q(i+1)-\frac{5}{128} E Q(i+2)
\end{aligned}
$$

Para o equalizador de cinco coeficientes, os pontos intermediários podem ser calculados como:

$$
\begin{gathered}
E Q 1=\frac{5}{243} E Q(i-3)-\frac{35}{243} E Q(i-2)+\frac{70}{81} E Q(i-1)+\frac{70}{243} E Q(i)-\frac{7}{243} E Q(i+1) \\
E Q 2=\frac{-7}{243} E Q(i-2)+\frac{70}{243} E Q(i-1)+\frac{70}{81} E Q(i)-\frac{35}{243} E Q(i+1)+\frac{5}{243} E Q(i+2) \\
E Q 3=\frac{5}{243} E Q(i-2)-\frac{35}{243} E Q(i-1)+\frac{70}{81} E Q(i)+\frac{70}{243} E Q(i+1)-\frac{7}{243} E Q(i+2) \\
E Q 4=\frac{-7}{243} E Q(i-1)+\frac{70}{243} E Q(i)+\frac{70}{81} E Q(i+1)-\frac{35}{243} E Q(i+2)+\frac{5}{243} E Q(i+3)
\end{gathered}
$$

Note que a equação (5) foi obtida utilizando $D=-1 / 2$ e a equação (6) utilizando $D=+1 / 2$. Entretanto, as equações (7) e (9) foram obtidas utilizando $D=+1 / 3$ e as equações (8) e (10) utilizando $D=-1 / 3$, devido às respectivas distâncias dos pontos em relação ao ponto central do subcanal. 
A Figura 5 mostra a resposta temporal dos interpoladores de Lagrange que realizam a interpolação no domínio da frequência, para diferentes números de coeficientes e para $D=1 / 2$. O efeito de filtragem passabaixa é claramente visível. A resposta ao impulso do canal deve ser menor do que o período do símbolo, e ela deve estar localizada na proximidade da origem do tempo.

A resposta ao impulso do canal Veicular A utilizado para comparar o desempenho dos equalizadores tem um comprimento de 2,6 $\mu \mathrm{s}$. Este comprimento da resposta ao impulso foi estimado para uma frequência de amostragem de $10 \mathrm{MHz}$. Então, da Figura 5, podemos concluir que para um sistema FBMC com 256 subcanais um interpolador de Lagrange de $5^{\text {a. }}$ ordem é suficiente para o canal Veicular A.

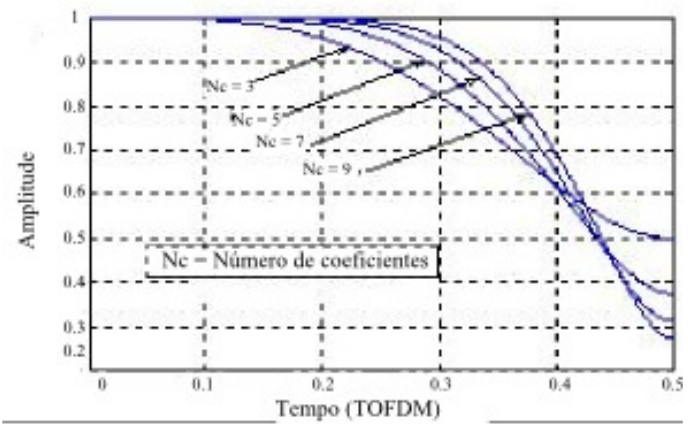

Fig. 5. Resposta temporal do interpolador de Lagrange no domínio da frequência para $\mathrm{D}=1 / 2$

O interpolador de Lagrange tem um bom desempenho se a duração da resposta ao impulso do canal é menor do que a duração da parte plana da resposta do interpolador no domínio do tempo. Em outras palavras, o desempenho do interpolador de Lagrange é fortemente dependente do offset temporal; este não é o caso do interpolador geométrico.

\section{B. Interpolação Geométrica}

A interpolação geométrica consiste em calcular a média geométrica de dois valores conhecidos. Ela é uma interpolação linear da fase e é praticamente linear para a amplitude, se os valores conhecidos de amplitude são próximos.

No caso presente, uma amostra intermediária da resposta em frequência do equalizador é dada por

$$
E Q i=X_{i}\left(X_{i \pm 1} / X_{i}\right)^{\rho}
$$

onde $X i$ é um ponto conhecido da resposta em frequência inversa do subcanal (por exemplo, $E Q(i-1), E Q(i)$ ou $E Q(i+1)$ na Figura 2), $X_{i+-1}$ são os pontos dos subcanais adjacentes conhecidos e $\rho$ é a fração da interpolação [11]. Para equalizadores de 3 e 5 coeficientes, $\rho$ corresponde a
$1 / 2$ e $1 / 3$, respectivamente. Os pontos intermediários para equalizadores de 3 coeficientes são calculados por

$$
E Q 1=E Q(i-1)(E Q(i) / E Q(i-1))^{1 / 2}
$$

$\mathrm{e}$

$$
E Q 2=E Q(i)(E Q(i+1) / E Q(i))^{1 / 2}
$$

Para os subcanais quasi-planos, os valores de $E Q(i-1)$, $E Q(i)$ e $E Q(i+1)$ são muito similares. Nestes casos, a magnitude de EQ2 na equação (13), por exemplo, pode ser aproximada por:

$$
|E Q 2| \approx \frac{1}{2}(|E Q(i)|+|E Q(i+1)|) .
$$

Portanto, a magnitude é quasi-linearmente interpolada. Evidentemente, para um canal plano tanto a magnitude quanto a fase devem ser linearmente interpoladas.

\section{Cálculo dos coeficientes usando a IFFT}

O uso da FFT e da IFFT para amostragem em frequência é uma técnica clássica utilizada em várias aplicações de processamento digital de sinais. Aqui usaremos a IFFT para calcular os coeficientes do equalizador de cada subcanal.

Iremos restringir a nossa análise a equalizadores com um número ímpar de coeficientes, calculados utilizando uma amostragem uniforme da resposta de frequência inversa do canal, como mostram as Figuras 3 e 4. Equalizadores com um número par de coeficientes apresentam desempenho pior porque os pontos conhecidos $E Q(i-n), n=. .-1,0,+1, \ldots$ não são utilizados para o cálculo dos coeficientes do equalizador.

Discutiremos apenas o projeto para o equalizador FIR de três coeficientes. O projeto para o equalizador FIR de cinco ou mais coeficientes pode ser feito seguindo o mesmo procedimento.

Os pontos de frequência $E Q(i \pm n), n=0,1,2, \ldots$ são obtidos utilizando um passo de frequência $\Delta f$ igual ao espaçamento dos subcanais. Na equalização FIR de três coeficientes utilizamos um ponto de frequência que está localizado no centro da banda do i-ésimo subcanal $(\omega=0)$, e duas frequências intermediárias, localizadas em $\omega= \pm \pi / 2$, como mostra a Figura 3. Os coeficientes do equalizador são calculados de forma que a resposta em frequência do equalizador passa pelos valores $E Q 1, E Q(i)$ e $E Q 2$. No banco de filtros de análise, a frequência de amostragem para cada subcanal é $2 \Delta f$. Como o espaçamento em frequência entre $E Q(i)$ e os pontos intermediários $E Q 1$ e $E Q 2$ é $\Delta f / 2$, teremos $2 \Delta f /(\Delta f / 2)=4$ pontos de frequência por subcanal. Então, um valor é desconhecido. Denotaremos por A o valor desconhecido. A pode ser encontrado por uma restrição sobre os coeficientes do equalizador calculados utilizando a IFFT de $4^{\text {a. }}$ ordem do conjunto $\{\boldsymbol{A}, E Q 1, E Q(i), E Q 2\}$. Considerando o i-ésimo subcanal, os coeficientes do equalizador obtidos depois da IFFT são $\left\{q_{0 i}, q_{1 i}, q_{2 i}, q_{3 i}\right\}$. O 
coeficiente central $q_{0 i}$ é circundado pelos coeficientes $q_{-1 i}=$ $q_{3 i}$ e $q_{1 i}$, devido à propriedade de periodicidade da IFFT. Considerando que queremos projetar um equalizador de 3 coeficientes, é necessário anular o coeficiente $q_{2 i}$; então podemos determinar o valor desconhecido A pela relação

$$
q_{2 i}=\frac{1}{4}(A-E Q 1+E Q(i)-E Q 2)=0 .
$$

Resolvendo para os outros coeficientes, temos:

$$
\begin{aligned}
& q_{-1 i}= \pm \frac{1}{4}(E Q 1-2 E Q(i)+E Q 2)+j(E Q 2-E Q 1) \\
& q_{0 i}= \pm \frac{1}{2}(E Q 1+E Q 2) \\
& q_{1 i}= \pm \frac{1}{4}(E Q 1-2 E Q(i)+E Q 2)-j(E Q 2-E Q 1)
\end{aligned}
$$

onde os sinais $-\mathrm{e}+$ correspondem aos subcanais pares e ímpares, respectivamente. Estas equações são similares às apresentadas em [1]. A maior diferença entre nossa técnica e a proposta em [1] é o grau de liberdade adicional dado pela variável A.

A Figura 6 resume a técnica proposta. Ela envolve dois passos principais: redução de ruído e derivação do valor dos coeficientes.

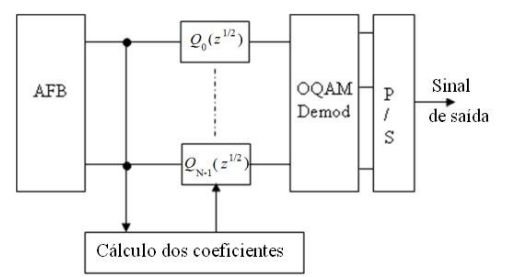

(a)

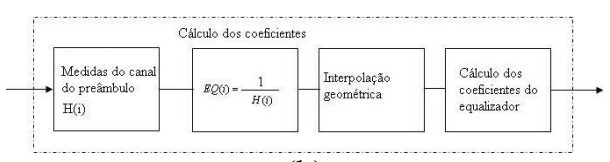

(b)

Fig.6 - A técnica de equalização por subcanal proposta

\section{Resultados DE Simulação}

Para comparar as diversas técnicas de projeto de equalizadores, o sistema FBMC/OQAM foi simulado utilizando o modelo de canal ruidoso quasi-estático Veicular A. Uma filtragem passa-baixa baseada na FFT e supondo o exato conhecimento do comprimento do canal foi utilizada para minorar o efeito do ruído na estimação do canal. Esta técnica de filtragem requer um par extra de FFTs e IFFTs.

Os parâmetros principais utilizados em nossas simulações foram:

1. Frequência de amostragem: $10 \mathrm{MHz}$

2. Frequência da portadora: $2,5 \mathrm{GHz}$

3. Número de subcanais: 128 e 256.

4. Número de realizações independentes do canal: 1000

\section{Comprimento do quadro: 53 símbolos OFDM}

6. Constelação OQPSK.

Como parâmetros de comparação, foram utilizados um equalizador MMSE e o sistema OFDM convencional. Os coeficientes do equalizador MMSE foram obtidos adaptativamente. A BER é calculada apenas depois da convergência dos coeficientes.

Foram realizadas simulações com o equalizador de cinco coeficientes, mas seu resultado mostrou-se idêntico ao do equalizador de três coeficientes.

A Figura 7 apresenta os resultados da BER para o canal Veicular A com 128 subcanais. Claramente, o equalizador de 1 coeficiente tem o pior desempenho, e o equalizador MMSE tem o melhor desempenho para uma razão Eb/N0 alta entre os sistemas FBMC/OQAM. Entretanto, o sistema OFDM apresenta um desempenho um pouco melhor.

Já para 256 subcanais todos os equalizadores FBMC/OQAM têm praticamente o mesmo desempenho, como mostra a Figura 8. Este resultado pode ser explicado pelo fato que os subcanais são quase planos; assim, um equalizador de um coeficiente é suficiente para compensar a distorção do canal. Novamente, o sistema OFDM tem o melhor desempenho entre os comparados.

Se introduzirmos um atraso de 96 amostras nos sistemas utilizando 256 subcanais, o desempenho dos sistemas OFDM e FBMC/OQAM utilizando um equalizador de um coeficiente cai vertiginosamente, como mostra a Figura 9. Tal efeito acontece devido à introdução pelo atraso de uma resposta em fase dependente da frequência no canal, transformando subcanais planos em subcanais seletivos em frequência. Os equalizadores de 3 coeficientes compensam melhor este efeito, com vantagem para o equalizador MMSE e o que usa a interpolação geométrica. A queda de desempenho no sistema OFDM foi devido a manutenção do comprimento do prefixo cíclico (CP) quando o atraso foi introduzido.

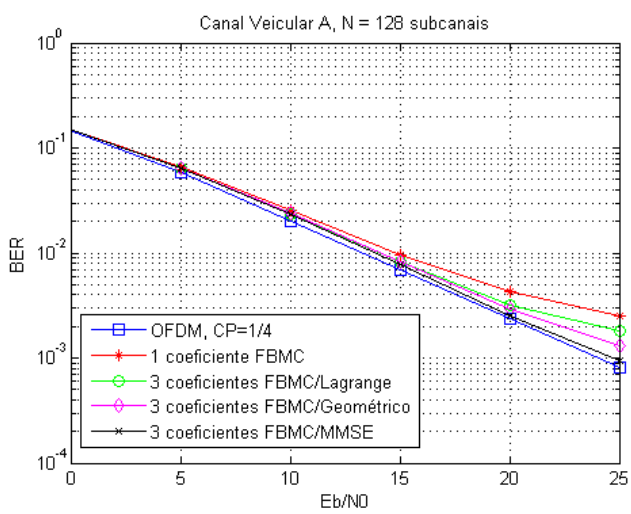

Fig. 7. Resultados de BER para diferentes equalizadores usando o canal Veicular A com 128 subcanais 


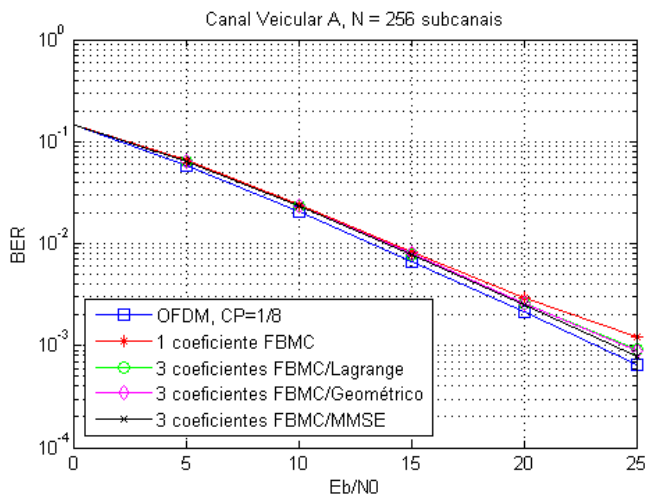

Fig. 8. Resultados de BER para diferentes equalizadores usando o canal Veicular A com 256 subcanais

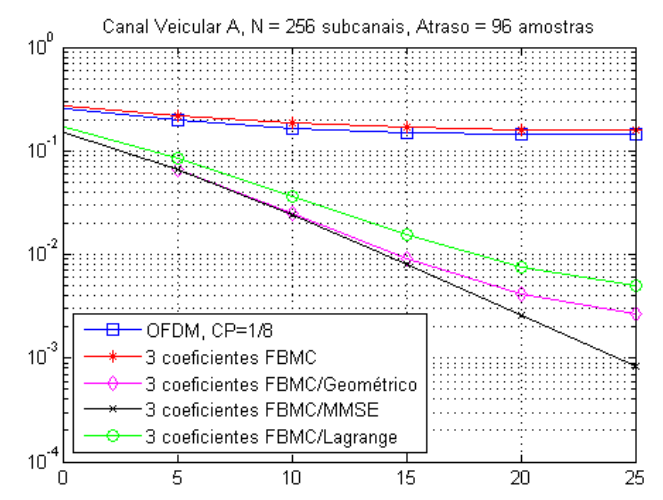

Fig. 9. Resultados de BER para diferentes equalizadores usando o canal Veicular A com 256 subcanais e atraso de 96 amostras

\section{CONCLUSÕES}

Neste artigo, propomos técnicas de projeto para equalizadores FIR de três e cinco coeficientes para sistemas de transmissão FBMC/OQAM baseados em amostragem de frequência e nos métodos de interpolação de Lagrange e geométrico. Foi assumido que o canal é invariante no tempo durante a duração dos bursts transmitidos.

Um equalizador MMSE foi considerado para comparar o desempenho dos equalizadores propostos. Foi demonstrado que um equalizador de um coeficiente apresenta desempenho similar aos equalizadores de três e cinco coeficientes para o canal veicular $\mathrm{A}$ e um alto número de subportadoras. Entretanto, quando um atraso é introduzido no sistema, os equalizadores com vários coeficientes apresentam melhor desempenho. Utilizando nossa proposta de técnica de projeto, os resultados de simulação demonstraram que o equalizador de três coeficientes tem o mesmo desempenho do de cinco coeficientes.
Devido à dependência da ordem do interpolador de Lagrange com a duração do canal, atrasos adicionais de transmissão deterioram o desempenho dos equalizadores baseados neste tipo de interpolação. $\mathrm{O}$ uso do interpolador geométrico torna o sistema mais robusto a esses atrasos.

\section{REFERÊNCIAS}

[1] T. Thalainen, T. Hidalgo Stitz, M. Rinne, and M. Renfors, "Channel Equalization in Filter Bank Based Multicarrier Modulation for Wireless Communications," EURASIP Journal on Advances in Signal Processing, vol. 2007, Article ID 49389, 18 pages, 2007.

[2] S.Nedic and N. Popovic, "Per-bin DFE for advanced OQAM-based multi-carrier wireless data transmission systems", Broadband Communications, 2002. Access, Transmission, Networking. 2002 International Zurich Seminaron, 2002 pp. 38-1 - 38-6

[3] D. S. Waldhauser, L. G. Baltar and J. A. Nossek, "MMSE subcarrier equalization for filter bank based multicarrier systems" In: 9th IEEE Workshop on Signal Processing Advances for Wireless Communications 2008. SPAWC 2008, Recife-Brazil.

[4] C. A. F. Rocha, M. Bellanger, 'Burst Transmission with FBMC/OQAM: Sub-Channel Equalizer Design', submetido ao IEEE International Symposium on Wireless Communication Systems 2009 [5] C. A. F. Rocha, M. Bellanger, Sub-Channel Equalizer Design Based on Geometric Interpolation for FBMC/OQAM Systems, submetido a International Conference on Signal Processing and Communication Systems 2009.

[6] ITU-R, "Guidelines for evaluation of radio transmission technologies for IMT-2000", Recommendation M.1225, 1997

[7] P. Siohan, C. Siclet, and N. Lacaille, Analysis and Design of OFDM/OQAM Systems Based on Filter Bank Theory, IEEE Transactions on Signal Processing, vol. 50, no. 5, pp. 1170-1183, 2002.

[8] H. Bölcskei, P. Duhamel, R. Hleiss: Orthogonalization of OFDM/OQAM Pulse Shaping Filters Using the Discrete Zak Transform, Signal Processing, vol.83(7), pp.1379-1391, 2003.

[9] M.G. Bellanger, "Specification and design of a prototype filter for filter bank based multicarrier transmission", IEEE International Conference on Acoustics, Speech, and Signal Processing, vol 4., pp. 2417-2420.

[10] M. Bellanger, "Traitement Numérique du Signal: Théorie et pratique", Dunod, Paris, 2008, 8 e édition.

[11] D. W. Gunness, Loudspeaker Transfer Function Averaging and Interpolation, 111th Convention of the Audio Engineering Society, September 2001 